Dear Author,

Please, note that changes made to the HTML content will be added to the article before publication, but are not reflected in this PDF.

Note also that this file should not be used for submitting corrections. 


\title{
Feasibility of a Chest-worn accelerometer for physical activity measurement
}

\author{
4 Q1 Joni H. Zhang, Duncan J. Macfarlane*, Tanja Sobko \\ Institute of Human Performance, The University of Hong Kong, Hong Kong
}

\section{A R T I C L E I N F O}

\section{Article history:}

Received 27 October 2015

Received in revised form 19 February 2016

Accepted 10 March 2016

Available online $\mathrm{xxx}$

\section{Keywords:}

Measurement

Activity monitors

Actigraph

Wrist worn

Treadmill

Objective monitoring

\section{A B S T R A C T}

Objectives: To examine the feasibility of wearing an Actigraph GT3X+ at the chest (resembling a neck pendant) for physical activity measurement.

Design: A convenience sample of 45 healthy adults (23 male, mean age $20.0 \pm 4.5$ years) was included in data analysis.

Methods: Participants simultaneously wore three GT3X+ accelerometers, on the Waist, Wrist, and Chest and completed 8 bouts of slow $(.67, .89,1.11 \mathrm{~m} / \mathrm{s})$, average $(1.33,1.56,1.78 \mathrm{~m} / \mathrm{s})$ and fast $(2.00,2.22 \mathrm{~m} / \mathrm{s})$ walking on a treadmill. Paired $t$-test, correlations and absolute percentage errors (APE) of accelerometer output (vector magnitude, VM) were computed for the key pairs: Waist-Wrist; and Waist-Chest. Results: The Wrist-site overestimated VM to a greater extent at all speeds in comparison to the Chest. Pearson's $r$ correlations were weaker for Waist-Wrist $(<.80)$ in comparison to the Waist-Chest $(>.85)$. The APE's were much lower (i.e. higher agreement) for the Chest (9.23-15.5\%) compared to the Wrist (19.7-54.9\%). Participants also felt the Chest-site was more acceptable than the Waist-site.

Conclusions: PA measurements recorded by a Chest worn GT3X+ more closely resembled PA measurements recorded at the traditional Waist site than when compared to the Wrist site. When combined with high Chest site preference, the findings of our study suggest that the Chest is a feasible site for accelerometer wear.

(C) 2016 Published by Elsevier Ltd on behalf of Sports Medicine Australia.

\section{Introduction}

There is an increasing use of wearable monitors in the objective assessment of physical activity (PA). Accelerometers have frequently been selected as the device-of-choice because of their small size and ability to characterize the intensity and duration of PA over long periods of time.

The traditional wear-site of an accelerometer is on the Waist, but this site has caused compliance issues. As low as $25 \%$ of participants provided the requested 7 days of data, citing reasons of discomfort or inconvenience of wearing a device on the waist over long periods. ${ }^{1}$ Similar problems were also found in children and youth studies. ${ }^{2-4}$ Consequently, when the "wrist-worn accelerometer" was adopted by the NHANES, participant compliance increased substantially from $40-70 \%$ in the 2003-2004 cycle (Waist-wear) to 70-80\% in the 2011-2012 cycle (Wrist-wear). However, the validity and reliability of a Wrist-worn device remains debatable. ${ }^{5}$ The

\footnotetext{
* Corresponding author.

E-mail address: djmac@hku.hk (D.J. Macfarlane).
}

NHANES attempted to overcome this limitation by using raw acceleration data and log transformations instead of activity counts in its data analyses. Yet data processing of Wrist accelerometry data remain challenging and much more complex than the Waist data because of the Wrist gesticulation and variability in movement, ${ }^{6}$ which often requires high computational costs and sophisticated analytic skills ${ }^{7}$ to achieve comparable accuracy to the Waist. This heightens the need for an alternative wear-site.

Recently, there has been a growing interest in Chest-worn accelerometers (directly attached to the participant's skin or via a chest-strap). Ian Cleland et $\mathrm{al}^{8}{ }^{8}$ showed that an accelerometer placed at the Chest performed better in correctly detecting daily activities using various machines learning algorithms than an accelerometer placed at the wrist. Moreover, Altini et al. ${ }^{9}$ showed that a single accelerometer at the Chest together with a combined activity-specific estimation method was very accurate for EE estimations. These studies have emphasized on the feasibility of a Chest-worn device in activity-specific measurements (i.e. posture recognition, fall detection) as opposed to levels of PA measurement. It is therefore worthwhile to investigate whether a Chest-worn accelerometer is applicable for the measurement of PA. 


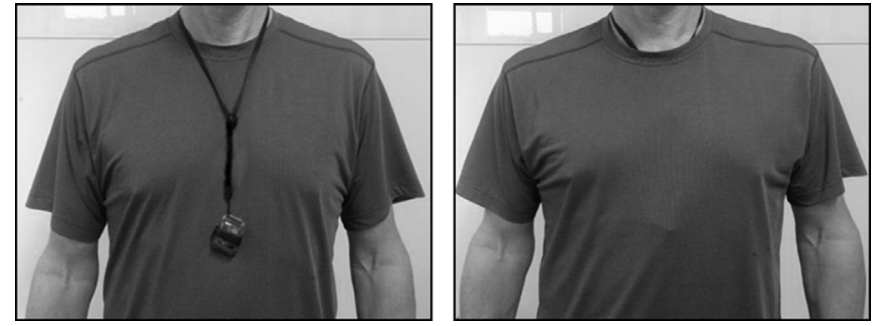

Fig. 1. Illustration of the Chest accelerometer necklace and positioning above and beneath (normal site) clothing.

The aim of this study was to determine whether participants would be more likely to agree to wear a commonly-used research accelerometer at the Chest, without significantly compromising measurement agreement, when compared to the traditional Waist and Wrist site. Thus this paper was a "proof-of-concept" study aimed at examining the feasibility of wearing the GT3X+ at the Chest for the measurement of PA by examining both the initial site preference, as well as the comparability of measurements made at the Chest, Wrist and Waist.

\section{Methods}

Participants were recruited from the neighbourhood of The University of Hong Kong by convenience sampling, including word of mouth and distribution of posters and leaflets. Inclusion criteria of the study were (1) apparently healthy adolescents/adults ( $\geq 15$ years but $<60$ years) with $\mathrm{BMI}<30 \mathrm{~kg} / \mathrm{m}^{2}$ and (2) individuals free of any type of walking aid/impediment. All participants read and signed an informed consent form, with the study protocol and ethics being approved by the Human Research Ethics Committee for Non-Clinical Faculties of the University of Hong Kong.

Testing was conducted in a laboratory at the Institute of Human Performance, the University of Hong Kong. Prior to testing, height $(\mathrm{m})$ and weight $(\mathrm{kg})$ of participants were measured using a stadiometer (Seca 213) and a Body Composition Analyzer (Tanita Model TBF-410), respectively. For height and weight measurements participants were asked to remove shoes, heavy clothing and belongings. The usual formula of body mass $(\mathrm{kg})$ divided by height squared $\left(\mathrm{m}^{2}\right)$ was used to compute the BMI for each participant.

To determine site preference, pictures of how each monitor is sited on the Waist, Wrist and around the neck (Chest) along with its corresponding accessory (Waist belt, Wrist strap and necklace) were first placed on a table in front of interviewees in no particular order. Interviewees were asked to rank the wear-sites according to how convenient and user-friendly they were perceived and prior to participation in the treadmill tests. For statistical analyses, a chi-square test of independence was performed to examine the relationship between wear-site and preference.

Upon completion of anthropometric measurements, instructions and assistance for simultaneously wearing three GT3X+ accelerometers on the Waist (located on the right waist in the mid-axillary line using a waistband), Wrist (located on the nondominant wrist with a wrist-strap) and Chest (located around the neck using a soft nylon necklace, length was adjusted to ensure the GT3X+ was near the xiphisternum underneath clothing, Fig. 1) were given to participants.

Participants performed 8 bouts of $\operatorname{slow}(.67, .89,1.11 \mathrm{~m} / \mathrm{s})$, average $(1.33,1.56,1.78 \mathrm{~m} / \mathrm{s})$ and fast $(2.00,2.22 \mathrm{~m} / \mathrm{s})$ walking/jogging on a preprogramed treadmill (Woodway, UK) in a controlled laboratory environment. Participants ambulated at each speed for 4-min with 1-minute break between each bout.

The Actigraph GT3X+ accelerometer (Actigraph, Pensacola, FL, USA) was used throughout, it is a lightweight (19g), small
$(4.6 \mathrm{~cm} \times 3.3 \mathrm{~cm} \times 1.5 \mathrm{~cm})$ tri-axial activity monitor that records dynamic accelerations in a magnitude range of $\pm 6 \mathrm{G}$ 's over extended periods of time. The device then quantifies the detected accelerations as an arbitrary unit called activity counts (a count is registered each time the magnitude of acceleration of the activity exceeds a given threshold) in three dimensions (axis $1=$ vertical, axis $2=$ horizontal right to left plane and axis $3=$ horizontal front to back plane). A summative score $\left(x^{2}+y^{2}+z^{2}\right)^{0.5}$ of the three axes is termed "vector magnitude (VM)", it is a common parameter for the estimation of PA (i.e. energy expenditure, activity intensity threshold) by capturing multidirectional movements. ${ }^{10}$ Therefore this parameter (VM) was selected as the outcome variable to be compared between the 3-sites. This approach is consistent with other recent studies aimed to examine intermonitor reliability of the GT3X+ accelerometers at hip, Wrist and Ankle sites during activities of daily living ${ }^{11}$ and to compare accelerometer output of different accelerometer models. ${ }^{12-14}$

All accelerometers were synchronized in time and initialized to collect data at a sampling rate of $100 \mathrm{~Hz}$. Data were downloaded using $1 \mathrm{~s}$ epochs and analyzed using Actilife version 6 software (Actigraph, Pensacola, USA). To compare monitor output, the first and forth minutes were removed and the middle portion of "matching" data were analyzed.

The statistical analyses were performed using SPSS version 20.0 (SPSS Inc., Chicago, IL) and MedCalc version 12.5 (MedCalc Software, Ostend, Belgium) for Windows. As the Waist-site is often considered as the traditional wear-site of accelerometers and has shown to be better at estimating energy expenditure ${ }^{15}$ (from its activity count output), as well as identifying activity intensity thresholds than the Wrist-site, ${ }^{16}$ it is therefore most logical to utilize measurements made at the Waist as the "referential criterion" for a proof-of-concept study as such. Hence all analyses were conducted for comparison pairs: Waist versus Wrist (Waist-Wrist), and Waist versus Chest (Waist-Chest) to examine comparability of measurements. Dependent $t$-tests and Cohen effect sizes $(d)^{17}$ were used to examine the size and magnitude of difference in VM scores between wear-sites at each speed bout. Pearson product-moment correlations were performed to quantify the relationship between VM scores recorded at each wear-site at each speed bout. Finally, absolute percentage errors (APE) were calculated for each comparison pair and Bland-Altman-type ${ }^{18}$ plots were generated using MedCalc to numerically and graphically illustrate the variability (magnitude and direction) of the error. All statistical tests were performed with a $p$-value of $<0.05$.

\section{Results}

Forty-five healthy young-adults (23 male) participated in the study (age: $20.0 \pm 4.5$ years; body mass index (BMI): $\left.20.5 \pm 2.9 \mathrm{~kg} / \mathrm{m}^{2}\right)$. All participants had complete accelerometer and questionnaire data.

Table 1 displays comparisons of VM scores. Across all speeds, statistically significant differences in VM scores were observed, with the absolute differences between the Waist-Wrist pair being much larger than the Waist-Chest pair. When compared to the Waist, statistically significant overestimation of VM at the Chest and Wrist were constantly observed with one exception, where the Chest significantly underestimated VM at slow walking speeds. The Cohen effect sizes for mean differences were considerably smaller (confirming higher agreement) for Chest-Waist comparison than the Chest-Wrist. Moreover, for average and fast walking speeds, Pearson's correlations were stronger for the Waist-Chest pair (>.85) compared to the Waist-Wrist pair (<.80). APE's were much lower (again confirming higher agreement with the Waist) for the Chest-site (9.23-15.5\%) when compared to the Wrist-site 
Table 1

Comparisons of VM recorded at the Wrist and Chest against the Waist.

\begin{tabular}{|c|c|c|c|c|c|}
\hline Walking condition $(\mathrm{m} / \mathrm{s})$ & Comparison with waist & Mean difference (SD) & APE (\%) & Effect size $(d)$ & Pearson's $r$ \\
\hline Slow & Wrist & $-899.9^{*}(2454.4)$ & 19.7 & 0.95 & $0.25^{*}$ \\
\hline$(.67-1.11)$ & Chest & $499.5^{*}(1238.2)$ & 10.9 & 0.73 & $0.64^{*}$ \\
\hline Average & Wrist & $-2644.6^{*}(3774.0)$ & 22.6 & 1.26 & $0.45^{*}$ \\
\hline$(1.33-1.78)$ & Chest & $-835.9^{*}(1502.4)$ & 9.23 & 0.51 & $0.85^{*}$ \\
\hline Fast & Wrist & $-21,212.7^{*}(12,565.9)$ & 54.9 & 1.40 & $0.73^{*}$ \\
\hline$(2.00-2.22)$ & Chest & $-3181.4(2965.3)$ & 15.5 & 0.65 & $0.91^{*}$ \\
\hline
\end{tabular}

Statistically significant at $p<0.05$.

(19.7-54.9\%). These findings were also diagrammatically supported by the modified Bland-Altman plots in Fig. 1, that show a markedly lower distribution of error scores (differences in VM) between Waist-Chest when compared to the Waist-Wrist for all speed conditions.

For questionnaire data, the chi-square test was significant $\chi^{2}$ $(4, N=45)=12.818, p<0.05$, indicating a significant difference in preference between sites. The observed frequency on ranks (first, second and third choice respectively), showed $22.7 \%, 27.3 \%$ and $50.0 \%$ selections for the Waist; $47.7 \%, 36.4 \%$ and $15.9 \%$ selections for the Wrist; with 29.5\%, 36.4\% and 34.1\% selections for the Chest. Overall, although the Chest-site was clearly more popular than the least-preferred Waist-site, it could not match the most preferred Wrist-site.

\section{Discussion}

Previous studies show that reasons for low compliance in accelerometry studies chiefly include inconvenience and physical discomfort of the wear-site (Waist/Hip), as well as negative feelings towards the appearance and visibility of wearing an accelerometer. ${ }^{1,2,4}$ Hence there is a need to identify alternative wear-sites that are able to enhance wear-compliance without significantly compromising agreement when compared to previously validated wear-sites. This is the first proof-of-concept study to investigate the feasibility of using the most common research-based accelerometer at a novel alternative site, the Chest, for the measurement of PA in a controlled treadmill condition. The feasibility was examined in two ways: (1) comparability of measurements recorded during locomotion and (2) comparing user preference, of the Waist-Chest and Waist-Wrist pairs.

The result of this study demonstrated that VM recorded at the Chest more closely resembles (i.e. higher comparability) the Waist, than the Wrist. This was consistently shown by the consistently higher agreements in VM scores, higher correlations and lower APE's between the Waist-Chest pair compared to the Waist-Wrist pair across all treadmill speeds. Furthermore, participants felt that the Chest-site was more acceptable than the traditional Waist-site. These findings, while preliminary, suggest that PA measurements made with a novel Chest/neck-worn GT3X+ not only closely resemble the traditional Waist-site, but also are likely to provide higher site preference than the Waist, thus striking a good balance between likely high compliance and acceptable criterion agreement. Additionally, it is important to note that, despite the Wrist-site being able to improve wear time and compliance, ${ }^{1}$ data analyses on Wrist data can be costly and complex, with Atallah et al., ${ }^{7}$ using 13 features extracted from the raw triaxial accelerometer signal to achieve an activity detection accuracy of $60-80 \%$, leading the authors to comment that their approach had high computational costs. Alternatively, our study has shown the Chest-site to be able to provide acceptably comparable accelerometer output when compared to the Waist-site, suggesting that similar analytical approach often used in the data analyses of Waist data could be applied to the Chest (i.e., lower cost, time and effort), and thus avoiding costly and complex data processing procedures used for the Wrist.

To date, this is the first study to examine the feasibility of the GT3X+ in worn at a novel Chest-site; consequently, direct comparisons with other studies are not possible, however, several other studies that examined less common devices (multisensory devices/accelerometers) appear to support the potential use of the Chest-site. One recent study ${ }^{9}$ examined how energy expenditure estimation error changed when based on the number of sensors and sensor positioning, using different combinations of Chest, Ankle, Thigh and Waist positions. These researchers used the gold standard of indirect calorimetry to validate the analysis and showed that the Chest sensor was the best single sensor site for energy expenditure estimation, whilst the Wrist sensor seemed to perform the worst. A second study ${ }^{8}$ compared the performance of six identical accelerometers (not commercially available) worn at the Chest, Wrist, Lower back, Hip, Thigh and Foot while participants undertook activities such as walking, sitting, lying, walking up and down stairs. It was found that the average percentage accuracy of an accelerometer worn at the Chest (96.91\%) was only marginally less than the most accurate Hip site (97.81\%), while the Wrist site was shown to be significantly less accurate (95.88). These findings, along with the results of our study provides justification for a novel GT3X+ wear location (Chest) to be proposed as a better alternative to existing wear-sites.

Furthermore, it has been suggested that estimating $\mathrm{EE}^{15}$ and identifying activity intensity thresholds ${ }^{16}$ from counts taken at the Wrist may introduce more error than the same calculations at the hip. This is because the large amount of gesticulation involving upper limbs does not correspond to large body movement (and therefore large EE). The proposed Chest-site is not prone to these types of limitations. It is also closer to the body's centre of mass, which is suggested to be ideal for accelerometer attachment. ${ }^{5}$ This phenomenon was diagrammatically illustrated by the Bland-Altman analyses (Fig. 2) in this study, where the distribution of Wrist error scores was considerably more scattered when compared to the Chest error scores. This finding is consistent with previous studies which explored the problem of detecting activities from Wrist-worn sensors. ${ }^{19,20}$ However the necklace attachment used for the Chest site may be prone to some pendulum-like movements that may be a source of potential noise, although our Chest site was specifically located under the clothing in order to contain this problem, but this warrants further investigation.

Although the novel Chest site showed very encouraging results, this study has several limitations. First, we delimited the study to a controlled laboratory setting and did not include a free-living condition using criterion measures such as the doubly-labelled water technique due to high costs. We felt that it was critical to begin our assessment of an Actigraph GT3X+ at this novel Chest-site by first examining its comparability to existing wear-sites, especially the traditional Waist-site (and secondly to the recently adopted Wrist site), by using highly controlled laboratory conditions designed to 

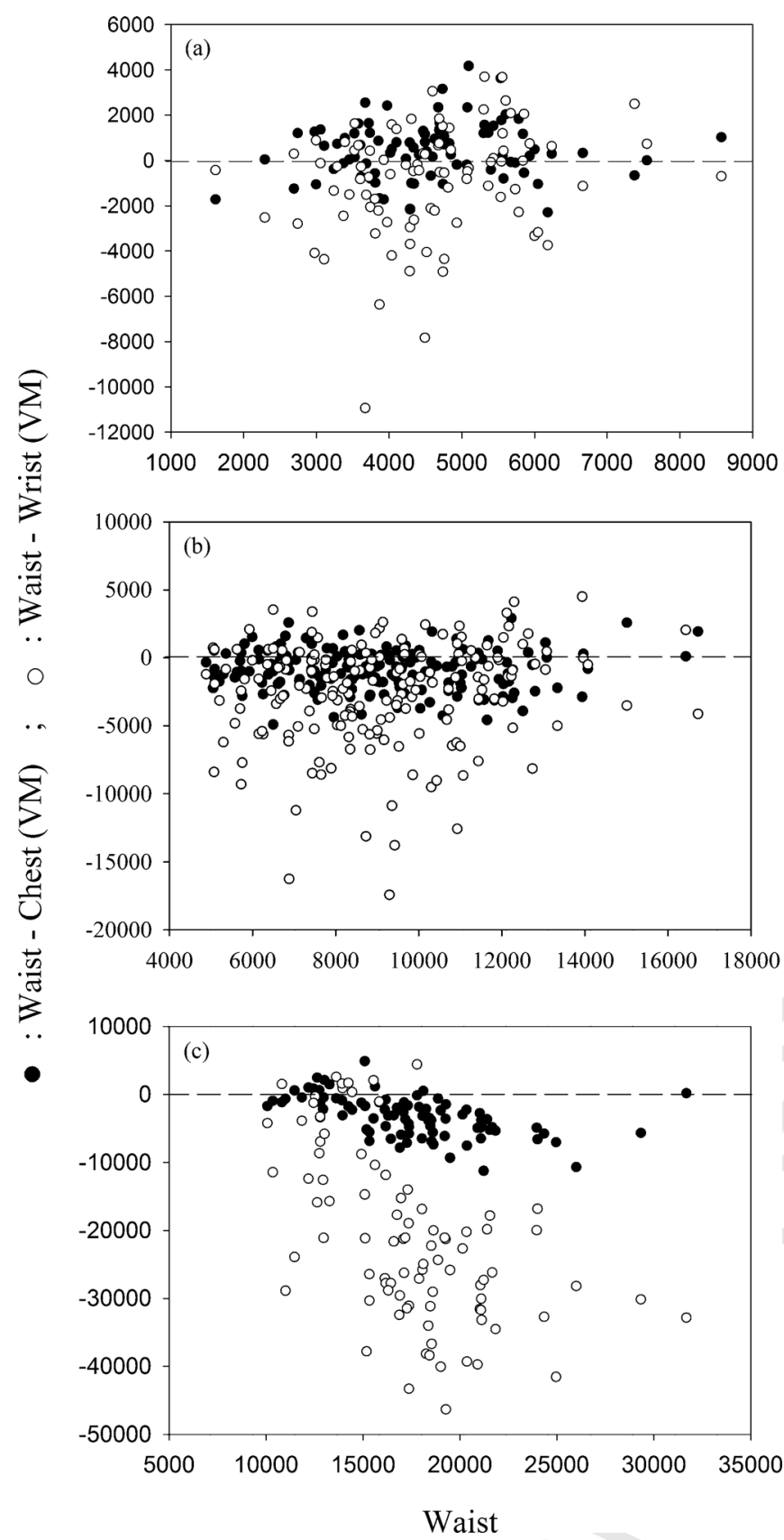

Fig. 2. Modified Bland-Altman plots showing distribution of error scores (difference in VM) of Waist-Wrist and Waist-Chest at (a) slow, (b) medium, and (c) fast walking.

have clearly defined incremental changes in accelerometer output. As this was only an exploratory "proof of concept" study that targeted the feasibility of the Chest site, no gas analysis was undertaken for energy expenditure/calibration analysis, as this would be done after feasibility was established. The second limitation of this study was the lack of simulated lifestyle activities within our laboratory setup. However, it has been suggested that locomotion is the primary mode by which most people accumulate light to moderate activity, therefore the findings of this exploratory study still adds significantly to the body of knowledge on accelerometry.

\section{Conclusions}

The PA measurements recorded using a common researchbased accelerometer at the novel Chest-site via a simple neck pendant more closely resembled PA measurements recorded at the traditional Waist site than when compared to the Wrist site. When combined with high Chest site preference, the findings of our study suggest that the Chest is a feasible site for accelerometer wear, especially for studies that wish to deploy activity monitors (accelerometers) for the assessment of PA levels. Future studies should aim at calibrating the Chest-site for the estimation of parameters such as EE and activity intensity thresholds. Because most international PA guidelines are based on the amount of time spent in moderate-to-vigorous physical activity (MVPA), we are currently conducting a calibration study to define intensity thresholds for moderate and vigorous physical activity for this novel Chest site.

\section{Practical implications}

- VM scores recorded by a Chest-worn accelerometer more closely resembles VM scores recorded by a Waist-worn accelerometer than the Wrist site.

- In the interests of increasing participant compliance without compromising on monitor accuracy, or the high costs and time in data processing for Wrist-site data, researchers are encouraged to consider the Chest-site for monitor-wear.

- Future calibration studies of the Chest-site are now required.

\section{Acknowledgements}

We would like to thank all the participants for their participation in the current study. All the authors declare no conflicts of interest. The present study was funded by the University of Hong Kong Seed Funding (EA180114).

\section{References}

Q4 317

1. Troiano RP, McClain JJ, Brychta RJ et al. Evolution of accelerometer methods for physical activity research. Br J Sports Med 2014; 48(13):1019-1023.

2. Audrey S, Bell S, Hughes R et al. Adolescent perspectives on wearing accelerometers to measure physical activity in population-based trials. Eur J Public Health 2012; 23(3):475-480

3. Crocker P, Holowachuk DR, Kowalski KC. Feasibility of using the Tritrac motion sensor over a 7-day trial with older children. Pediatr Exerc Sci 2001; 13(1):70-81.

4. Robertson W, Stewart-Brown S, Wilcock E et al. Utility of accelerometers to measure physical activity in children attending an obesity treatment intervention. $J$ Obes 2011:2011.

5. Trost SG, Mclver KL, Pate RR. Conducting accelerometer-based activity assessments in field-based research. Med Sci Sports Exerc 2005; 37(11):S531-S543.

6. Mannini A, Intille SS, Rosenberger $M$ et al. Activity recognition using a single accelerometer placed at the wrist or ankle. Med Sci Sports Exerc 2013; 45(11):2193-2203.

7. Atallah L, Lo B, King R et al. Sensor positioning for activity recognition using wearable accelerometers. IEEE Trans Biomed Circuits Syst 2011; 5(4):320-329.

8. Cleland I, Kikhia B, Nugent C et al. Optimal placement of accelerometers for the detection of everyday activities. Sensors 2013; 13(7):9183-9200.

9. Altini M, Penders J, Vullers R et al. Estimating energy expenditure using body-worn accelerometers: a comparison of methods, sensors number and positioning. IEEE J Biomed Health Inform 2014; 19(1):219-226.

10. Sasaki JE, John D, Freedson PS. Validation and comparison of ActiGraph activity monitors. J Sci Med Sport 2011; 14(5):411-416.

11. Ozemek C, Kirschner MM, Wilkerson BS et al. Intermonitor reliability of the GT3X+ accelerometer at hip, wrist and ankle sites during activities of daily living. Physiol Meas 2014; 35(2):129.

12. John D, Sasaki J, Staudenmayer J et al. Comparison of raw acceleration from the GENEA and ActiGraph ${ }^{\mathrm{TM}}$ GT3X+ activity monitors. Sensors 2013; 13(11):14754-14763.

13. Robusto KM, Trost SG. Comparison of three generations of ActiGraph ${ }^{\mathrm{TM}}$ activity monitors in children and adolescents. J Sports Sci 2012; 30(13):1429-1435.

14. Lee K-Y, Macfarlane DJ, Cerin E. Comparison of three models of actigraph accelerometers during free living and controlled laboratory conditions. Eur $J$ Sport Sci 2013; 13(3):332-339.

15. Rosenberger ME, Haskell WL, Albinali F et al. Estimating activity and sedentary behavior from an accelerometer on the hip or wrist. Med Sci Sports Exerc 2013; 45(5):964-975.

16. Tudor-Locke C, Barreira TV, Schuna Jr JM. Comparison of step outputs for waist and wrist accelerometer attachment sites. Med Sci Sports Exerc 2015; 47(4):839-842. 
17. Cohen J. The concepts of power analysis, In: Statistical Power Analysis for the Behavioral Sciences. 2nd ed. Hillsdale, NJ, Lawrence Earlbaum Associates, 1988 [Chapter 1].

18. Krouwer JS. Why Bland-Altman plots should use $\mathrm{X}$, not $(\mathrm{Y}+\mathrm{X}) / 2$ when $\mathrm{X}$ is a reference method. Stat Med 2008; 27(5):778-780.
19. Chen KY, Acra SA, Majchrzak K et al. Predicting energy expenditure of physical activity using hip-and wrist-worn accelerometers. Diabetes Technol Ther 2003; 5(6):1023-1033.

20. Zhang S, Rowlands A, Murray P et al. Physical activity classification using the GENEA wrist-worn accelerometer. Med Sci Sports Exerc 2012; 44(4):742-748.

Please cite this article in press as: Zhang JH, et al. Feasibility of a Chest-worn accelerometer for physical activity measurement. J Sci Med Sport (2016), http://dx.doi.org/10.1016/j.jsams.2016.03.004 